\title{
Dynein axonemal heavy chain 9 M4374I variation may have an effect on imatinib mesylate resistance in CML
}

\author{
MAHMUT SELMAN YILDIRIM ${ }^{1}$, LEVENT ŞIMSSEK ${ }^{1}$, AYŞE GÜL ZAMANi ${ }^{1}$, \\ ÖZCAN ÇENELİ ${ }^{2}$ and SİNAN DEMIRCIOĞLU ${ }^{2}$ \\ Departments of ${ }^{1}$ Medical Genetics, and ${ }^{2}$ Hematology, Meram Medical School, \\ Necmettin Erbakan University, Konya 42080, Turkey
}

Received October 18, 2021; Accepted January 17, 2022

DOI: $10.3892 / \mathrm{mi} .2022 .29$

\begin{abstract}
Chronic myelogenous leukemia (CML) is a myeloproliferative neoplasm caused by a translocation between the breakpoint cluster region (BCR) and Abelson murine leukemia 1 (ABL1) genes. Tyrosine kinase inhibitors (TKIs) are used in the treatment of CML. TKIs, bind the ABL1 kinase domain of hybrid BCR-ABL1 protein and inhibit its function. However, resistance can occur due to the pathogenic variations in the ABL kinase domain or BCR-ABL1-independent mechanisms. In the present study, genetic variations possibly related to imatinib resistance in CML were explored. A total of five single nucleotide polymorphisms [SNPs; MORN2 rs3099950, PTCRA rs9471966, ANKRD35 rs11579366, dynein axonemal heavy chain 9 (DNAH9) rs1990236 and MAGEC1 rs176037] were investigated in imatinib sensitive and in resistant CML patients. Additionally, sequencing of the ABL1 kinase domain was also performed. The frequency of DNAH9 M4374I (NP_001363.2)/M686I (NP_004653.2) (rs1990236) was found to be significantly higher in the imatinib-resistant group. However, the other SNPs did not exhibit any statistically significant differences and no new variant was detected in the ABL1 kinase domain. Considering the frequency difference of the DNAH9 rs1990236 between imatinib-sensitive and imatinib-resistant groups, DNAH9 gene may play a role in TKI resistance. Due to the limited amounts of literature available on this subject, further studies on DNAH9 and related genes may prove to be beneficial for the elucidation of the association between DNAH9 and TKI resistance. Moreover, further larger studies are required to support the current findings. This
\end{abstract}

Correspondence to: Professor Mahmut Selman Yıldırım, Department of Medical Genetics, Meram Medical School, Necmettin Erbakan University, 281 Beyşehir Avenue, Akyokuş, Konya 42080, Turkey

E-mail: drmselman@hotmail.com

Key words: dynein axonemal heavy chain 9, chronic myelogenous leukemia, imatinib resistance, tyrosine kinase inhibitors, Philadelphia chromosome may aid in the development of novel treatment protocols for patients with CML with DNAH9 genetic polymorphisms.

\section{Introduction}

Chronic myeloid leukemia (CML) is a myeloproliferative neoplasm characterized by an abnormal proliferation of myeloid cells in bone marrow and accounts for approximately $15 \%$ of all adult leukemias (1). CML is the first type of cancer in which an underlying genetic mechanism was identified, and pathogenesis-specific targeted therapy can be successfully applied. The Philadelphia $(\mathrm{Ph})$ chromosome originated from reciprocal translocation between the Abelson murine leukemia 1 (ABL1) gene on chromosome 9 (9q34) and the breakpoint cluster region (BCR) gene on chromosome 22 (22q11), is responsible for the pathogenesis of CML. The Ph chromosome is detected in $95 \%$ of cases. The product of this translocation, the BCR-ABL1 fusion protein, is responsible for cellular differentiation in myeloid cells with irregular and excessive tyrosine kinase activity, leading to increased proliferation and the inhibition of apoptosis (2).

Tyrosine kinase inhibitors (TKIs) and resistance development. Currently, tyrosine kinase protein-specific inhibitory drugs have been developed and are successfully used for the treatment of CML. The first of these drugs is imatinib mesylate. Imatinib binds to the ABL1 kinase domain of the BCR-ABL1 fusion protein and inhibits tyrosine kinase activity. After the discovery of this drug, a significant increase in the survival expectancy and quality of life of patients with CML was observed (3).

Over the years, the addition of imatinib to CML therapy has increased the 5-year survival rate of patients with $\mathrm{CML}$ from 22 to $69 \%$ (4). However, $25 \%$ of patients do not benefit from imatinib treatment (primary resistance) (5).

Response to TKI treatment is assessed hematologically and molecularly. The hematological response is defined by the normalization of the blood count and spleen size. On the other hand, the molecular response is measured by the BCR-ABL1 transcript levels calculated on the International Scale (IS) (6). Reverse transcription-quantitative polymerase chain reaction (RT-qPCR) analysis is routinely used for the calculation of transcript levels. Patients with BCR-ABL1 transcript levels 
$<10 \%$ after 3 months, $<1 \%$ after 6 months or $<0.1 \%$ after 12 months are considered molecularly to be optimally responsive to treatment. However, BCR-ABL1 transcript levels $>10 \%$ after 3 or 6 months or $>1 \%$ after 12 months are considered as a treatment failure (7).

Furthermore, $\sim 20-25 \%$ of patients who benefit from the treatment exhibit resistance to imatinib therapy after the complete hematological or molecular response (acquired resistance) (8). The re-increase in BCR-ABL1 activity is generally known as the cause for acquired resistance in CML. The increase in BCR-ABL1 activity may occur due to the amplification of the fusion gene, an increased expression, or mutations of the ABL kinase sequence of the fusion gene. Mutations of the ABL kinase domain are the most frequent mechanisms of TKI resistance, particularly for the acquired type (9). However, not all mechanisms of resistance are dependent on BCR-ABL1; for instance, the alternative activation of mTOR has been shown to be a BCR-ABL1-independent mechanism for resistance (10). Another study demonstrated that Fas/FasL signaling pathway polymorphisms also have an effect on imatinib response (11). In the literature, variations in different regions of the genome other than the BCR-ABL1 fusion gene, have been shown to be associated with TKI resistance (11-14). The detection of these genetic variations may enable the prediction of prognosis and efficacy of TKIs beforehand. Moreover, studies aimed at discovering these variations may pave the way for combined therapies that target multiple pathways in addition to BCR-ABL1 (15). In one of these studies, Lavrov et al (16) determined several candidate single nucleotide polymorphisms (SNPs) to predict TKI resistance in patients with CML. In that study article, it was stated that the SNPs of the MORN repeat containing 2 (MORN2) gene (rs3099950), Pre T-cell antigen receptor alpha (PTCRA) gene (rs9471966), ankyrin repeat domain 35 (ANKRD35) gene (rs11579366), dynein axonemal heavy chain 9 (DNAH9) gene (rs1990236) and the MAGE family member C1 (MAGEC1) gene (rs176037) may have a high predictive value.

In the present study, exons 4, 5 and 6 of the ABL1 gene and SNPs in the MORN2, PTCRA, ANKRD35, DNAH9 and MAGEC1 genes were analyzed in imatinib mesylate-sensitive and -resistant patients with CML. By sequencing the ABL1 kinase domain, the authors aimed to detect new variations in the ABL1 gene responsible for BCR-ABL1-dependent resistance. In addition, by determining the SNP frequencies of the MORN2, PTCRA, ANKRD35, DNAH9 and MAGEC1 genes to reveal the possible mechanisms related to BCR-ABL1-independent resistance, the authors hope to contribute to the development of novel treatment strategies for CML.

\section{Patients and methods}

The present study was conducted in the Hematology and Medical Genetics Departments of Necmettin Erbakan University Meram Medical School Hospital and received approval from Necmettin Erbakan University Meram Medical School Ethics Committee and informed written consent for participation and publication were obtained from the patients (protocol no. 2018/1539).
Patient selection. Imatinib-sensitive and imatinib-resistant groups were formed from the patients with CML who visited the Hematology Outpatient Clinic of Necmettin Erbakan University Meram Medical School Hospital. Patients with CML on imatinib therapy who were followed-up for at least 1 year and were in remission were included in the imatinib-sensitive group. Patients who had the following criteria were included in the imatinib-resistant group: i) No early molecular response at 3-6 months of imatinib therapy; ii) no major molecular response to imatinib in the first year of treatment; and iii) no response to imatinib and follow-up with 2 nd and 3rd generation TKIs.

Patients with any of the following criteria were excluded from the study: i) Newly diagnosed, untreated or treated for $<1$ year; ii) failure to comply with the drug therapy or follow-ups; iii) harboring a known pathogenic variant in the ABL1 kinase domain that was previously associated with TKI resistance.

A total of 131 patients with CML followed-by Hematology and Medical Genetics departments of Necmettin Erbakan University Meram Medical School Hospital for at least 1 year were evaluated. In total, 74 of these patients were imatinib-sensitive and 57 of them were imatinib-resistant. Of the imatinib-sensitive patients, 50 were selected according to their demographic characteristics to form the imatinib-sensitive group. In addition, 9 patients out of the 57 imatinib-resistant patients were excluded as they did not meet the inclusion criteria. Of these patients, 1 patient was excluded due to non-compliance at follow-up, 3 patients due to intolerable side-effects of imatinib, and 5 patients due to a known pathogenic variant in the ABL1 kinase domain. Of the 5 patients with a pathogenic variant in the ABL1 kinase domain, 3 were positive for T315I, 1 patient was positive for F317L, and 1 patient was positive for $\mathrm{Y} 253 \mathrm{H}$. After excluding the patients who did not meet the inclusion criteria, the imatinib-resistant group had 48 patients. All patients in both groups were Caucasian.

A total of 20 of the imatinib-resistant patients were under nilotinib therapy, 22 of them were being treated with dasatinib, 4 patients were being treated with ponatinib, and 2 patients with bosutinib. The female/male ratios for the imatinib-resistant and imatinib-sensitive groups were 1.4 (28 females and 20 males) and 2.3 (35 females and 15 males), respectively.

Karyotyping and fluorescence in situ hybridization (FISH). Upon admission, for each patient, karyotyping and FISH analyses were performed on the bone marrow aspirates. Metaphases, obtained from bone marrow cell culture, were banded using G-banding and imaged (Lucia Cytogenetics 1.5.6 software, Lucia Cytogenetics-Karyo) and analyzed using standards asserted by the International System for Human Cytogenetic Nomenclature (ISCN 2016) (17). FISH using BCR-ABL1 dual color fusion probe (LPH 007, Cytocell) was applied to the interphases obtained from bone marrow aspirates of the patients. Bone marrow aspirate $(\sim 1 \mathrm{ml})$ was added to a solution containing $5 \mathrm{ml}$ RPMI-1640 medium (cat. no. 11875101, Thermo Fisher Scientific, Inc.) and $0.05 \mathrm{ml}$ penicillin-streptomycin $(5,000 \mathrm{IU}$ penicillin, $5,000 \mu \mathrm{g} / \mathrm{ml}$ streptomycin; 450-200-EL, Wisent Bioproducts). Subsequently, $0.5 \mathrm{ml}$ Colcemid solution $(10 \mu \mathrm{g} / \mathrm{ml} \mathrm{N}$-deacetyl- $\mathrm{N}$-methyl 
colchicine in Dulbecco's phosphate-buffered saline; 12-004-1D, Biological Industries) was added to the sample and incubated at $37^{\circ} \mathrm{C}$ for $30 \mathrm{~min}$. Following incubation, the sample was centrifuged at $400 \mathrm{x}$ g for $6 \mathrm{~min}$. The supernatant was then discarded and the remaining sample was vortexed. A total of $10 \mathrm{ml}$ hypotonic solution $(5.7 \mathrm{~g}$ potassium chloride dissolved in 1 liter distilled water) warmed to $37^{\circ} \mathrm{C}$ and added to the sample. Subsequently, the sample was incubated at $37^{\circ} \mathrm{C}$ for $30 \mathrm{~min}$ and was then centrifuged at $400 \mathrm{x} \mathrm{g}$ for $6 \mathrm{~min}$. Following centrifugation, the supernatant was discarded and the remaining sample vortexed and $10 \mathrm{ml}$ fixative solution (Carnoy's solution: 3:1 methanol/acetic acid; Supelco, Merck $\mathrm{KGaA})$ at $-18^{\circ} \mathrm{C}$ was added to the sample. The last three steps were repeated twice with 5 and $3 \mathrm{ml}$ fixative solutions, respectively. The sample was then placed onto a glass microscope slide and allowed to dry. The slide was then immersed in $2 \mathrm{X}$ saline-sodium citrate (SSC; $17.5 \mathrm{~g} \mathrm{NaCl}$ and $8.8 \mathrm{~g}$ sodium citrate dissolved in 1 liter distilled water) for $2 \mathrm{~min}$ at room temperature. Subsequently, the slide was placed in an ethanol series (70, 85 and 100\%), each for $2 \mathrm{~min}$ at room temperature and then allowed to dry. After this step, $10 \mu \mathrm{l}$ hybridization buffer (LPH 007, Cytocell) containing probes pre-mixed in hybridization solution (formamide; dextran sulphate; (SSC), added onto the sample slide at $37^{\circ} \mathrm{C}$. For denaturation, the slide was placed on a hotplate at $75^{\circ} \mathrm{C}$ for $2 \mathrm{~min}$.

For the hybridization step, the slide incubated in a humid, lightproof container at $37^{\circ} \mathrm{C}$ for $16 \mathrm{~h}$. Following hybridization, the slide was placed in $0.4 \mathrm{X}$ SSC (5-fold diluted $2 \mathrm{X} \mathrm{SSC}$ ) at $72^{\circ} \mathrm{C}$ for $2 \mathrm{~min}$. Subsequently, the slide was immersed in $2 \mathrm{X}$ SSC, $0.05 \%$ Tween-20 (polysorbate 20) at room temperature for $30 \mathrm{sec}$ and $10 \mu \mathrm{l}$ 4',6-diamidino-2-phenylindole (DAPI) antifade $(0.125 \mu \mathrm{g} / \mathrm{ml}$ DAPI; DES 500L, Cytocell) was added onto the slide. After this step, the slide was covered with a coverslip, and incubated in the dark at room temperature for $10 \mathrm{~min}$. Afterwards, each slide was analyzed under a fluorescent microscope (Eclipse 80i, Nikon). A minimum of 100 interphases were analyzed for each patient.

$R T-q P C R$. RNA isolation was conducted using the Hybrid- $\mathrm{R}^{\mathrm{TM}}$ Blood RNA kit (Geneall Biotechnology Co. Ltd.) from the venous blood of patients. Subsequently, quantitative PCR (qPCR) was performed using the isolated RNA with the geneMAP $^{\text {тм }}$ BCR-ABL1 P210 (GenMark Diagnostics) on the LightCycler ${ }^{\circledR} 480$ system (Roche Diagnostics). The PCR cycling conditions are presented in Table I. The BCR-ABL1/ABL1 ratio was calculated according to the IS (6).

DNA isolation. Subsequently, the venous blood was collected from the patients into EDTA tubes, barcoded and stored immediately at $-20^{\circ} \mathrm{C}$. DNA extraction was performed from these samples using the Roche High Pure PCR Template kit (Roche Diagnostics). The purity of the DNA was measured using a Thermo Scientific Nanodrop Spectrophotometer (Thermo Fisher Scientific, Inc.). The 260/280 and 260/230 nm nucleic acid purity ratios were in the normal range (1.7-2.0 and 2.0-2.2, respectively) for all samples.

SNP genotyping. qPCR was performed to determine genotype of the patients for the SNPs. The extracted DNA was mixed with WizPure ${ }^{\mathrm{TM}}$ qPCR Master UDG (probe) solution
Table I. Protocol for BCR-ABL1 RT-qPCR.

\begin{tabular}{lccc}
\hline Step & Temperature $\left({ }^{\circ} \mathrm{C}\right)$ & Duration & No. of cycles \\
\hline $\begin{array}{l}\text { Contamination } \\
\text { prevention }\end{array}$ & 50 & $30 \mathrm{~min}$ & 1 \\
$\begin{array}{l}\text { Polymerase } \\
\text { activation }\end{array}$ & 95 & $15 \mathrm{~min}$ & 1 \\
$\begin{array}{l}\text { Denaturation } \\
\text { Annealing/ } \\
\text { extension }\end{array}$ & 95 & $15 \mathrm{sec}$ & 45 \\
\hline
\end{tabular}

Table II. PCR protocol for SNP genotyping.

\begin{tabular}{lccc}
\hline Step & Temperature $\left({ }^{\circ} \mathrm{C}\right)$ & Duration & No. of cycles \\
\hline $\begin{array}{l}\text { Polymerase } \\
\text { activation }\end{array}$ & 95 & $10 \mathrm{~min}$ & 1 \\
$\begin{array}{l}\text { Denaturation } \\
\text { Annealing/ }\end{array}$ & 95 & $15 \mathrm{sec}$ & 40 \\
extension & 60 & $1 \mathrm{~min}$ & \\
\hline
\end{tabular}

(Wizbiosolutions, Inc.) and Applied Biosystems ${ }^{\mathrm{TM}}$ TaqMan $^{\mathrm{TM}}$ SNP Genotyping Assay (cat. no. 4351379; Applied Biosystems; Thermo Fisher Scientific, Inc.) containing the primers for MORN2 rs3099950, PTCRA rs9471966, ANKRD35 rs11579366, DNAH9 rs1990236 and MAGEC1 rs176037 SNPs. This mix was then loaded onto the Applied Biosystems ${ }^{\mathrm{TM}} 7500$ fast system (Applied Biosystems; Thermo Fisher Scientific, Inc.) and the PCR protocol presented in Table II was used. Genotypes of each SNP were determined by analyzing signals derived from VIC ${ }^{\mathrm{TM}}$ and FAM ${ }^{\mathrm{TM}}$ labeled alleles using TaqMan ${ }^{\circledR}$ Genotyper Software (Applied Biosystems; Thermo Fisher Scientific, Inc.).

Sanger sequencing. For sequencing of the ABL kinase domain, primers for exons 4, 5 and 6 of the ABL1 gene were designed using the NCBI Primer Designing Tool (https:/www.ncbi. nlm.nih.gov/tools/primer-blast/). The sequences of the primers are presented in Table III. Sanger sequencing was conducted to isolated DNAs of all patients with an Applied Biosystems 3500 Genetic Analyzer (Applied Biosystems; Thermo Fisher Scientific, Inc.).

Bioinformatic analysis. SPSS Statistics 26.0 software (IBM Corp.) and the SNPStats web tool for SNP analysis (18) were used for statistical analyses.

Statistical analysis. The exact test for Hardy-Weinberg equilibrium in the SNPStats tool (18) was used for the calculation of deviation from Hardy-Weinberg equilibrium separately for each SNP in both groups $(n=98)$. Univariate logistic regression analysis was used for association analysis between five SNPs (MORN2 rs3099950, PTCRA rs9471966, ANKRD35 rs11579366, DNAH9 rs1990236 and MAGEC1 rs176037) and imatinib resistance with different genetic models $(n=98)$. These 
Table III. Sequences of primers for the ABL1 gene.

Location and direction

of primer

Sequence of primer
Exon 4 forward

Exon 5 forward

Exon 5 reverse

Exon 6 forward

Exon 6 reverse
Exon 4 reverse
AGCTCTTTGAGCTTGCCTGT GATGCATCGCCTAATGCCAG GTATGCGCTGAAGCTCCATTT TCCAACGAGGTTTTGTGCAG TGCTTGGGACCATGTTGGAA CCTAGGCTGGGGCTTTTTGT models were codominant, dominant, recessive, over-dominant and log-additive. In addition, the frequency of each SNP was compared between the imatinib-sensitive and imatinib-resistant groups using Fisher's exact test $(n=98)$. Moreover, odds ratios (ORs) and 95\% confidence intervals (95\% CIs) for the analyses of the SNPs were also calculated. A P-value $<0.05$ was considered to indicate a statistically significant difference for all of the analyses mentioned above $(\alpha=0.05)$.

The Sanger sequencing results of the ABL1 kinase domain were also analyzed. Since there was no variant compared to the reference sequence in both groups, no statistical analysis was performed.

\section{Results}

Upon patient diagnosis, karyotyping, FISH and RT-qPCR were conducted for all patients. The Ph chromosome in karyotype analysis (Fig. 1), and BCR-ABL1 fusion in FISH (Fig. 2) were detected in all patients. In addition, $>10 \%$ BCR-ABL1 (IS) levels in RT-qPCR (Fig. 3) were also detected in all patients within both groups.

Sanger sequencing was performed on exons 4,5 and 6 of the ABL1 gene and revealed no differences between the imatinib-sensitive and imatinib-resistant groups. There was no variation in the sequenced regions compared to the reference sequence.

The majority of the SNPs exhibited Hardy-Weinberg equilibrium within both groups. However, PTCRA rs9471966 and MAGEC1 rs176037 were in disequilibrium in the imatinib-resistant and imatinib-sensitive groups, respectively (Table IV). However, the allelic frequencies of both SNPs were similar between the sensitive and resistant groups (P-values: 0.562 and 0.253 respectively) (Table $\mathrm{V}$ ).

For DNAH9 rs1990236 (c.13122G>A), 25 of the 48 patients in the imatinib-resistant group (52.1\%) were homozygous wild-type (GG), 16 patients $(33.3 \%)$ were heterozygous (GA) and 7 patients (14.6\%) were homozygous (AA) for this SNP. However, in the imatinib-sensitive group, 38 of the 50 patients $(76.0 \%)$ were homozygous wild-type (GG), 10 $(20.0 \%)$ were heterozygous (GA) and 2 of them (4.0\%) were homozygous (AA) for DNAH9 rs1990236. The frequency of DNAH9 rs1990236 (c.13122G>A) was significantly higher in the imatinib-resistant group with an $\mathrm{OR}$ of $2.792(\mathrm{P}=0.003$; Table V).

For MORN2 rs3099950 (c.142G>A), 39 of the $48(81.2 \%)$ patients in the imatinib-resistant group were homozygous wild-type (GG), 8 patients $(16.7 \%)$ were heterozygous (GA) and 1 patient $(2.0 \%)$ was homozygous (AA). On the other hand, in the imatinib-sensitive group, 38 of the 50 patients $(76.0 \%)$ were homozygous wild-type (GG), 12 (24.0\%) were heterozygous (GA) and there was no homozygous (AA) patient for this variant. The frequencies of MORN2 rs3099950 (c.142G>A) between the imatinib-resistant and imatinib-sensitive groups exhibited no statistically significant difference (OR, 0.853; $\mathrm{P}=0.451$; Table V).

For PTCRA rs9471966 (c.316G>A), 30 of the $48(62.5 \%)$ patients in the imatinib-resistant group were homozygous wild-type (GG), 11 patients $(22.9 \%)$ were heterozygous (GA) and 7 patients (14.6\%) were homozygous (AA) for this SNP. On the other hand, 28 of the 50 patients $(56.0 \%)$ in the imatinib-sensitive group were homozygous wild-type (GG), $18(36.0 \%)$ were heterozygous (GA) and 4 patients $(8.0 \%)$ were homozygous (AA). Between the imatinib-resistant and imatinib-sensitive groups, the frequencies of PTCRA rs9471966 (c.316G>A) exhibited no statistically significant difference (OR, 1.002; $\mathrm{P}=0.562$; Table V).

For ANKRD35 rs11579366 (c.1981G>C), 11 of the 48 $(22.9 \%)$ patients in the imatinib-resistant group were homozygous wild-type (GG), 26 patients $(54.2 \%)$ were heterozygous (GC) and 11 patients (22.9\%) were homozygous (CC) for this SNP. However, in the imatinib-sensitive group, 9 of the 50 patients $(18.0 \%)$ were homozygous wild-type (GG), 27 $(54.0 \%)$ were heterozygous (GC) and 14 (28.0\%) were homozygous (CC) for this variant. The frequencies of ANKRD35 rs11579366 (c.1981G>C) between the imatinib-resistant and imatinib-sensitive groups did not exhibit any statistically significant difference (OR, 0.818; $\mathrm{P}=0.289$; Table V).

Furthermore, 21 of the $48(43.7 \%)$ imatinib-resistant patients were homozygous wild-type (CC) for MAGEC1 rs176037 (c.452C>T), 21 of them $(43.7 \%)$ were heterozygous (CT) and 6 of them (12.5\%) were homozygous (TT) for this SNP. On the other hand, in the imatinib-sensitive group, 22 of the 50 patients $(44.0 \%)$ were homozygous wild-type (CC), $16(32.0 \%)$ were heterozygous (CT) and 12 (24.0\%) were homozygous (TT) for this SNP. The frequencies of MAGEC1 rs176037 (c.452C>T) between the imatinib-resistant and imatinib-sensitive groups did not exhibit any statistically significant difference (OR, 0.786; $\mathrm{P}=0.253$; Table V).

The detailed frequencies of MORN2 rs3099950, PTCRA rs9471966, ANKRD35 rs11579366, DNAH9 rs1990236 and MAGEC1 rs176037 SNPs among the imatinib-sensitive and imatinib-resistant groups are presented in Table V.

\section{Discussion}

Imatinib is a good early example of targeted cancer therapy and has markedly improved the survival of patients with CML over the years (4). However, resistance to imatinib and other TKIs is a major issue for CML therapy, rendering the detection and prediction of resistance more crucial. Depending on the development mechanisms, resistance to TKIs can be divided into two categories, BCR-ABL1-dependent and -independent resistance (19). The overexpression of BCR-ABL1 and mutations in the ABL1 kinase domain are main BCR-ABL1-dependent resistance mechanisms. On the other hand, BCR-ABL1-independent resistance can be 

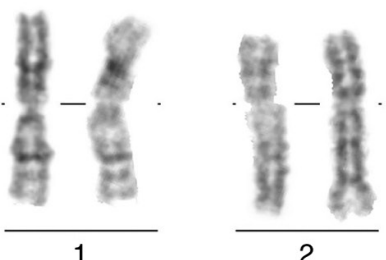

2
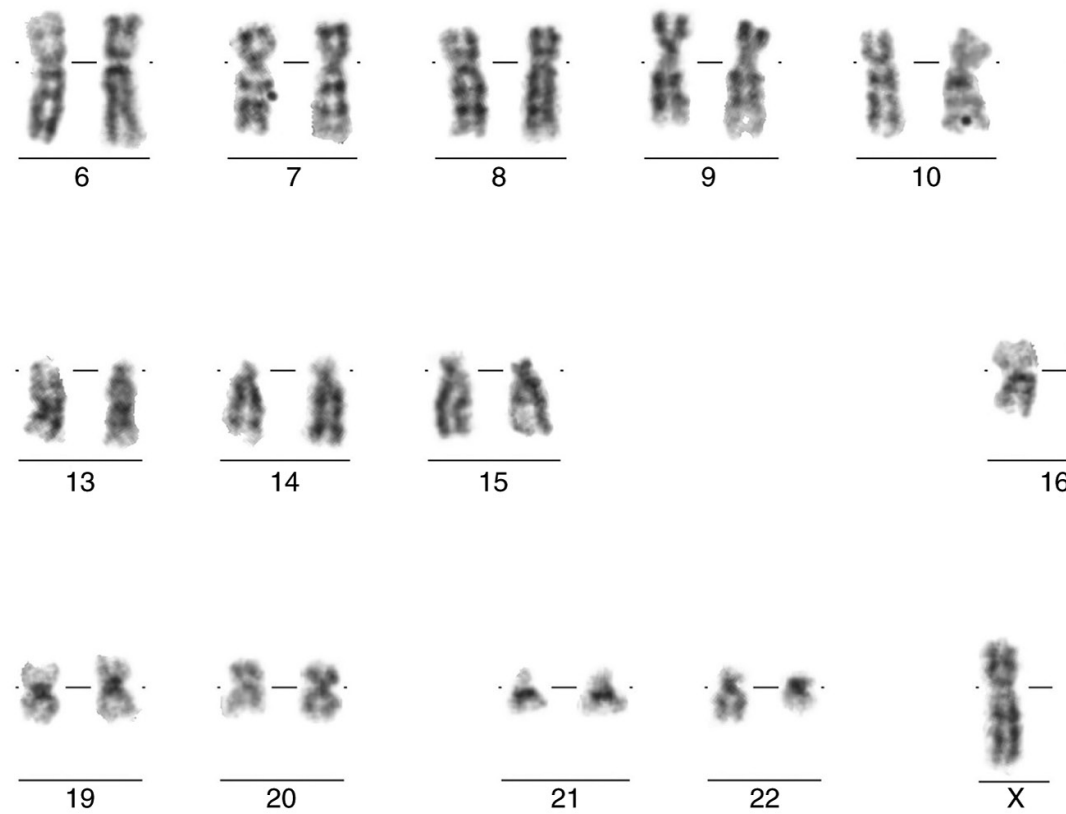
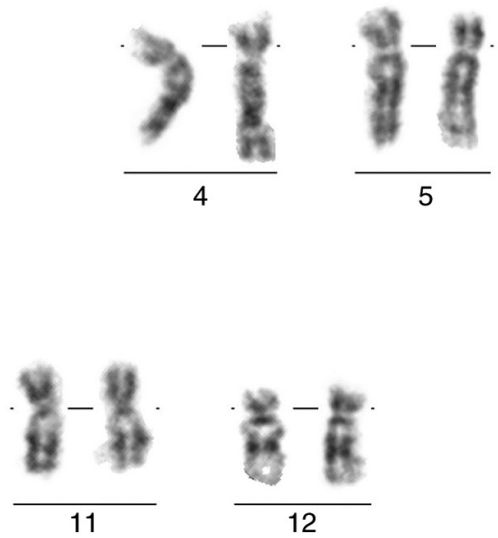

Figure 1. Karyotype of one of the patients exhibiting a translocation between chromosome 9 and 22 .

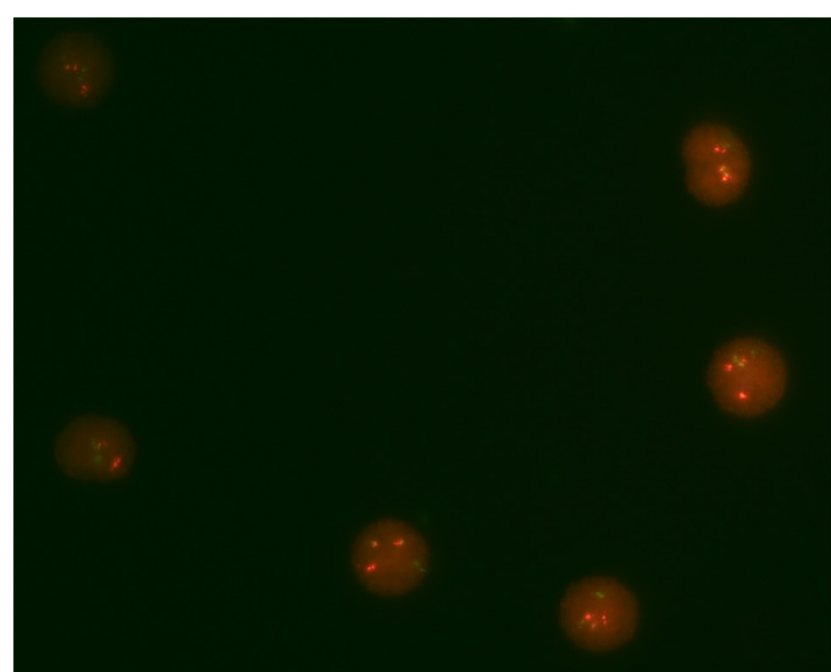

Figure 2. Interphase FISH analysis of one of the patients exhibiting dual fusions of $\mathrm{t}(9 ; 22)$ in multiple interphases.

caused by a number of mechanisms, such as pharmacokinetic factors, clonal evolution, or disruption of signaling pathways $(10,11,15,19)$. Despite these known contributing mechanisms, BCR-ABL1-independent resistance remains 'terra incognita', encompassing promising areas of research.
The present study investigated five SNPs and three exons of the ABL1 kinase domain of the BCR-ABL1 gene. It was found that the frequency of the DNAH9 rs1990236 allele was significantly higher in the resistant group than in the sensitive group.

The DNAH9 gene is chromosomally located in the $17 \mathrm{p} 12$ region. It encodes the heavy chain of the axonemal dynein protein. Dyneins are multimeric microtubule-associated motor proteins and provide cells or intracellular components with motility. The product of the DNAH9 gene joins type two outer dynein arms, which are essential for cilia and flagella movement (20). According to the Genotype-Tissue Expression (GTEx) database (https://gtexportal.org/home/), tissues in which DNAH9 is extensively expressed are the testes, fallopian tubes, brain and lungs (21).

Homozygous germline pathogenic variations of DNAH9 are associated with primary ciliary dyskinesia. Pathogenic variations of DNAH9 are also detected in esophageal squamous cell carcinoma, triple-negative breast cancer and invasive micropapillary carcinomas of the breast (22-24). Moreover, a somatic DNAH9 variant (c.10242+5G>A) has been reported in a patient with atypical CML (25). Another study demonstrated the abnormal methylation of DNAH9 in non-small cell lung cancer (26). In addition, a study on circulating tumor DNA of patients with hepatocellular carcinoma using next-generation sequencing revealed DNAH9 variations in $32 \%$ of patients (27). Next-generation sequencing technologies can provide massive amounts of data on thousands of 
Table IV. Deviation analysis for Hardy-Weinberg equilibrium ${ }^{\mathrm{a}}$.

\begin{tabular}{lccccccc}
\hline SNP & Group & $\begin{array}{c}\text { Homozygous } \\
\text { wild-type }\end{array}$ & $\begin{array}{c}\text { Heterozygous } \\
\text { wild-type }\end{array}$ & $\begin{array}{c}\text { Homozygous } \\
\text { variant }\end{array}$ & $\begin{array}{c}\text { Wild-type allele } \\
\text { frequency }\end{array}$ & $\begin{array}{c}\text { Variant allele } \\
\text { frequency }\end{array}$ & P-value \\
\hline MORN2 (rs3099950) & IS & 38 & 12 & 0 & 88 & 12 & 0.98 \\
G $>$ A & IR & 39 & 8 & 1 & 86 & 10 & 0.41 \\
PTCRA (rs9471966) & IS & 28 & 18 & 4 & 74 & 26 & 0.71 \\
G $>$ A & IR & 30 & 11 & 7 & 71 & 25 & 0.0071 \\
ANKRD35 & IS & 14 & 27 & 9 & 55 & 45 & 0.77 \\
(rs11579366) G>C & IR & 11 & 26 & 11 & 48 & 48 & 0.77 \\
DNAH9 (rs1990236) & IS & 38 & 10 & 2 & 86 & 14 & 0.22 \\
G $>$ A & IR & 25 & 16 & 7 & 66 & 30 & 0.17 \\
MAGEC1 (rs176037) & IS & 22 & 16 & 12 & 60 & 40 & 0.02 \\
C $>$ T & IR & 21 & 21 & 6 & 63 & 33 & 0.99 \\
\hline
\end{tabular}

${ }^{a}$ The Exact test for Hardy-Weinberg equilibrium in the SNPStats tool (18). IS, imatinib-sensitive; IR, imatinib-resistant.
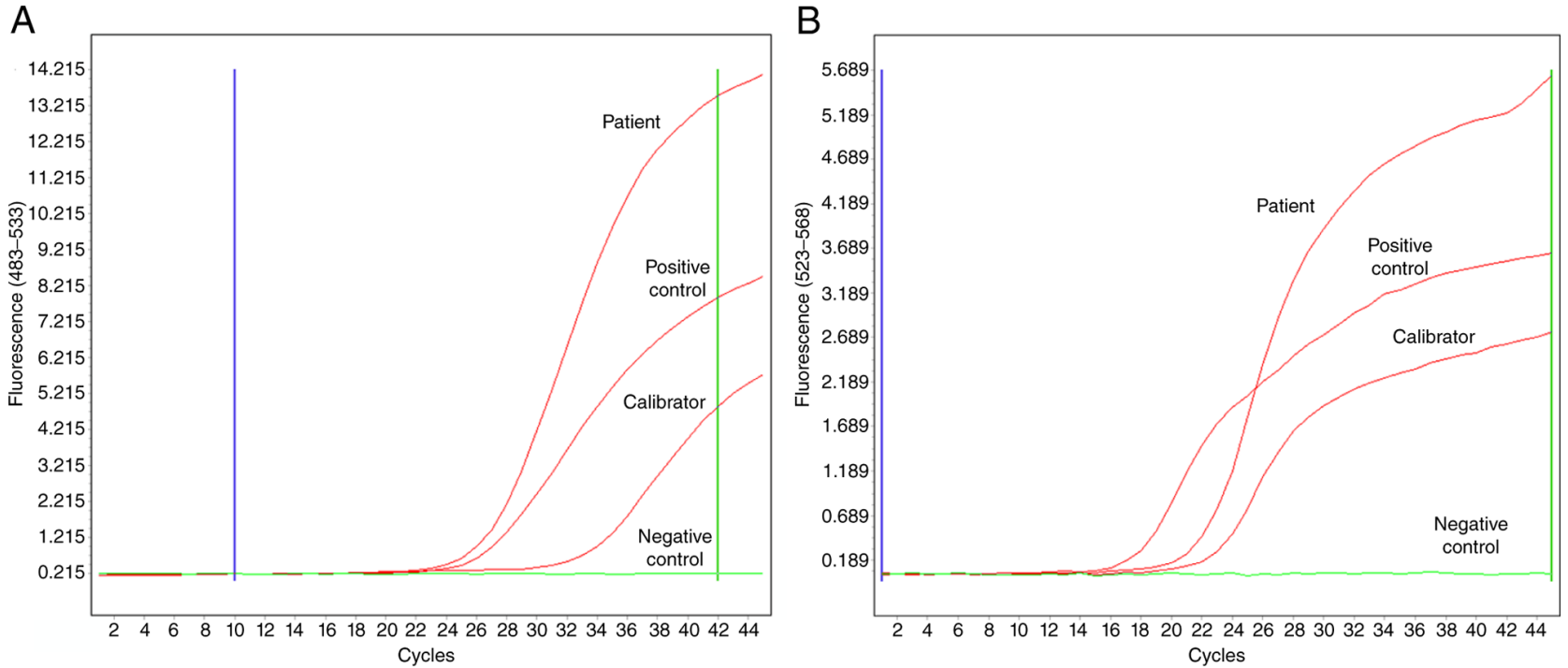

Figure 3. BCR-ABL1/ABL1 RT-qPCR analysis results of one of the patients using LightCycler ${ }^{\circledR} 480$ Software (Roche Diagnostics). Amplification curves in FAM $^{\mathrm{TM}}$ (A) and $\mathrm{VIC}^{\mathrm{TM}}$ (B) channels depicting BCR-ABL1 fusion and ABL1 transcript levels, respectively.

genes simultaneously. The use of these technologies on genes of unknown actionability, such as DNAH9, may reveal novel diagnostic, prognostic and therapeutic biomarkers for cancer patients in the future.

The variant DNAH9 c.13122G $>$ A [p.Met4374Ile (NP_001363.2)/p.Met686Ile (NP_004653.2)] investigated herein is a missense variant that causes the conversion of amino acid 4374 of the encoded protein from methionine to isoleucine. The frequency of this variant in the gnomAD genome database (https://gnomad.broadinstitute.org/) is 0.175 (28). In silico analyses to estimate pathogenicity revealed a DANN score of 0.9685 and a SIFT score of 0.021 , which were considered disruptive to protein function $(29,30)$.

Although the frequency of the DNAH9 rs1990236 allele in the present study was higher in the imatinib-resistant group, the frequency of this SNP was higher in the TKI-sensitive group in the study by Lavrov et al (16). In another study on 62 patients, there was no significant difference observed in
DNAH9 rs1990236 between optimal and non-optimal TKI responsive groups (31).

The discrepancy between these results may be attributed to the different group designs. In the studies by Lavrov et al $(16,31)$, responses to all TKIs were taken into account in the process of forming groups. In the present study, the response to a specific TKI (imatinib) was selected as the variable for group classification.

There is no known direct association between DNAH9 and imatinib resistance in CML demonstrated in the literature; however, the possible mechanisms can be hypothesized. For instance, the Sonic hedgehog pathway is involved in the survival of leukemic stem cells in CML, and the primary cilium plays a central role in the Sonic hedgehog pathway $(32,33)$. Although it is known that axonemal dyneins, such as DNAH9 do not participate in the primary cilium structure, the non-axonemal functions of DNAH9 may play a role in this regard. For example, GAS11, a dynein regulatory protein that has functions 
Table V. Frequencies of the five SNPs in the imatinib-resistant and imatinib-sensitive groups.

\begin{tabular}{|c|c|c|c|c|c|c|}
\hline Gene/SNP & Genotype & Imatinib-sensitive & Imatinib-resistant & OR & $95 \% \mathrm{CI}$ & P-value \\
\hline \multicolumn{7}{|c|}{ MORN2 (rs3099950) } \\
\hline \multicolumn{7}{|l|}{ Alleles ${ }^{\mathrm{a}}$} \\
\hline & $\mathrm{G}$ & $88(88 \%)$ & $86(89.6 \%)$ & Ref & & \\
\hline & $\mathrm{A}$ & $12(12 \%)$ & $10(10.4 \%)$ & 0.853 & $0.350-2.077$ & 0.451 \\
\hline \multicolumn{7}{|l|}{ Genetic models ${ }^{\mathrm{b}}$} \\
\hline \multirow[t]{3}{*}{ Codominant } & $\mathrm{G} / \mathrm{G}$ & $38(76 \%)$ & $39(81.2 \%)$ & Ref & & 0.34 \\
\hline & $\mathrm{G} / \mathrm{A}$ & $12(24 \%)$ & $8(16.7 \%)$ & 0.65 & $(0.24-1.77)$ & \\
\hline & $\mathrm{A} / \mathrm{A}$ & $0(0 \%)$ & $1(2.1 \%)$ & NA & $(0.00-\mathrm{NA})$ & \\
\hline \multirow[t]{2}{*}{ Dominant } & $\mathrm{G} / \mathrm{G}$ & $38(76 \%)$ & $39(81.2 \%)$ & Ref & & 0.53 \\
\hline & G/A-A/A & $12(24 \%)$ & $9(18.8 \%)$ & 0.73 & $(0.28-1.93)$ & \\
\hline \multirow[t]{2}{*}{ Recessive } & G/G-G/A & $50(100 \%)$ & $47(97.9 \%)$ & Ref & & 0.23 \\
\hline & $\mathrm{A} / \mathrm{A}$ & $0(0 \%)$ & $1(2.1 \%)$ & NA & (0.00-NA) & \\
\hline \multirow[t]{2}{*}{ Over-dominant } & $\mathrm{G} / \mathrm{G}-\mathrm{A} / \mathrm{A}$ & $38(76 \%)$ & $40(83.3 \%)$ & 1.00 & & 0.37 \\
\hline & $\mathrm{G} / \mathrm{A}$ & $12(24 \%)$ & $8(16.7 \%)$ & 0.63 & $(0.23-1.72)$ & \\
\hline Log-additive & - & - & - & 0.85 & $(0.34-2.09)$ & 0.72 \\
\hline
\end{tabular}

PTCRA (rs9471966)

Alleles ${ }^{\mathrm{a}}$

Genetic models ${ }^{\mathrm{b}}$

Codominant

G $74(74 \%)$

$71(73.9 \%)$

Ref

A $26(26 \%)$

$25(26 \%)$

1.002

0.529-1.897

0.562

$30(62.5 \%)$

Ref

$11(22.9 \%)$

0.57

$7(14.6 \%)$

1.63

$30(62.5 \%)$

Ref

$18(37.5 \%)$

0.76

$41(85.4 \%)$

$7(14.6 \%)$

Ref

$4(8 \%)$

Over-dominant

$32(64 \%)$

$37(77.1 \%)$

1.96

G/G-A/A

$18(36 \%)$

Ref

0.53

1.00

(0.23-1.42)

(0.43-6.19)

0.28

Log-additive

$\mathrm{G} / \mathrm{A}$

$11(22.9 \%)$

(0.34-1.71)

0.51

0.3

(0.54-7.19)

0.15

(0.22-1.28)

(0.56-1.78)

0.99

ANKRD35 (rs11579366)

Alleles $^{\mathrm{a}}$

$\begin{array}{ll}\mathrm{G} & 45(45 \%) \\ \mathrm{C} & 55(55 \%)\end{array}$

Genetic models ${ }^{\mathrm{b}}$

Codominant

$\mathrm{G} / \mathrm{G} \quad 9(18 \%)$

$\mathrm{G} / \mathrm{C}$

$\mathrm{C} / \mathrm{C}$

$27(54 \%)$

$14(28 \%)$

Dominant

Recessive

Over-dominant

Log-additive

DNAH9 (rs1990236)

Alleles ${ }^{\mathrm{a}}$

$\mathrm{G} / \mathrm{G} \quad 9(18 \%)$

$\mathrm{G} / \mathrm{C}-\mathrm{C} / \mathrm{C}$

$\mathrm{G} / \mathrm{G}-\mathrm{G} / \mathrm{C}$

$\mathrm{C} / \mathrm{C}$

G/G-C/C

$\mathrm{G} / \mathrm{C}$

$41(82 \%)$

$36(72 \%)$

$14(28 \%)$

$23(46 \%)$

$27(54 \%)$
$48(50 \%)$

$48(50 \%)$

$11(22.9 \%)$

$26(54.2 \%)$

$11(22.9 \%)$

$11(22.9 \%)$

$37(77.1 \%)$

$37(77.1 \%)$

$11(22.9 \%)$

$22(45.8 \%)$

$26(54.2 \%)$
Ref

0.818

0.467-1.435

0.289

0.76

$0.79 \quad(0.28-2.21)$

$0.64 \quad(0.20-2.10)$

Ref

0.74

Ref

0.76

Ref

1.01

0.80

(0.28-1.98)

0.55

0.56

(0.31-1.91)

0.99

(0.45-2.23)

(0.45-1.45) $\quad 0.46$

$\begin{array}{ll}\mathrm{G} & 86(86.0 \%) \\ \mathrm{A} & 14(14.0 \%)\end{array}$


Table V. Continued.

\begin{tabular}{|c|c|c|c|c|c|c|}
\hline Gene/SNP & Genotype & Imatinib-sensitive & Imatinib-resistant & OR & $95 \% \mathrm{CI}$ & P-value \\
\hline \multicolumn{7}{|l|}{ Genetic models ${ }^{\mathrm{b}}$} \\
\hline \multirow{3}{*}{ Codominant } & GG & $38(76.0 \%)$ & $25(52.1 \%)$ & Ref & & 0.03 \\
\hline & GA & $10(20.0 \%)$ & $16(33.3 \%)$ & 2.43 & $(0.95-6.21)$ & \\
\hline & $\mathrm{AA}$ & $2(4.0)$ & 7 (14.6) & 5.32 & $(1.02-27.72)$ & \\
\hline \multirow[t]{2}{*}{ Dominant } & $\mathrm{G} / \mathrm{G}$ & $38(76 \%)$ & $25(52.1 \%)$ & Ref & & 0.013 \\
\hline & $\mathrm{G} / \mathrm{A}-\mathrm{A} / \mathrm{A}$ & $12(24 \%)$ & $23(47.9 \%)$ & 2.91 & $(1.23-6.89)$ & \\
\hline \multirow[t]{2}{*}{ Recessive } & G/G-G/A & $48(96 \%)$ & $41(85.4 \%)$ & Ref & & 0.063 \\
\hline & $\mathrm{A} / \mathrm{A}$ & $2(4 \%)$ & $7(14.6 \%)$ & 4.10 & $(0.81-0.83)$ & \\
\hline \multirow[t]{2}{*}{ Over-dominant } & G/G-A/A & $40(80 \%)$ & $32(66.7 \%)$ & Ref & & 0.13 \\
\hline & G/A & $10(20 \%)$ & $16(33.3 \%)$ & 2.00 & $(0.80-5.00)$ & \\
\hline Log-additive & - & - & - & 2.36 & $(1.21-4.62)$ & 0.0082 \\
\hline \multicolumn{7}{|c|}{ MAGEC1 (rs176037) } \\
\hline \multicolumn{7}{|c|}{ Alleles $^{\mathrm{a}}$} \\
\hline & $\mathrm{C}$ & $60(60 \%)$ & $63(65.6 \%)$ & Ref & & \\
\hline & $\mathrm{T}$ & $40(40 \%)$ & $33(34.4 \%)$ & 0.786 & $0.440-1.405$ & 0.253 \\
\hline \multicolumn{7}{|l|}{ Genetic models ${ }^{b}$} \\
\hline \multirow[t]{3}{*}{ Codominant } & $\mathrm{C} / \mathrm{C}$ & $22(44 \%)$ & $21(43.8 \%)$ & Ref & & 0.26 \\
\hline & $\mathrm{C} / \mathrm{T}$ & $16(32 \%)$ & $21(43.8 \%)$ & 1.37 & $(0.57-3.33)$ & \\
\hline & $\mathrm{T} / \mathrm{T}$ & $12(24 \%)$ & $6(12.5 \%)$ & 0.52 & $(0.17-1.65)$ & \\
\hline \multirow[t]{2}{*}{ Dominant } & $\mathrm{C} / \mathrm{C}$ & $22(44 \%)$ & $21(43.8 \%)$ & Ref & & 0.98 \\
\hline & $\mathrm{C} / \mathrm{T}-\mathrm{T} / \mathrm{T}$ & $28(56 \%)$ & $27(56.2 \%)$ & 1.01 & $(0.45-2.24)$ & \\
\hline \multirow[t]{2}{*}{ Recessive } & $\mathrm{C} / \mathrm{C}-\mathrm{C} / \mathrm{T}$ & $38(76 \%)$ & $42(87.5 \%)$ & Ref & & 0.14 \\
\hline & $\mathrm{T} / \mathrm{T}$ & $12(24 \%)$ & $6(12.5 \%)$ & 0.45 & $(0.15-1.32)$ & \\
\hline \multirow[t]{2}{*}{ Over-dominant } & $\mathrm{C} / \mathrm{C}-\mathrm{T} / \mathrm{T}$ & $34(68 \%)$ & $27(56.2 \%)$ & Ref & & 0.23 \\
\hline & $\mathrm{C} / \mathrm{T}$ & $16(32 \%)$ & $21(43.8 \%)$ & 1.65 & $(0.73-3.77)$ & \\
\hline Log-additive & - & - & - & 0.82 & $(0.48-1.39)$ & 0.46 \\
\hline
\end{tabular}

a Data were analyzed using Fisher's exact test; ${ }^{b}$ univariate logistic regression analysis. OR, odds ratio; 95\% CI, 95\% confidence interval; Ref, reference allele.

primarily in motile cilia, has been shown to have additional non-axonemal functions. In addition, GAS11 is localized at the primary cilium base of kidney cells (34). Thus, DNAH9 may play a role in the survival of leukemic stem cells through the primary cilium. For an example of non-axonemal functions of DNAH9, it has been reported in the literature that DNAH9 interacts with BCL6, an important gene in the development of lymphoma (35). Since there is no comprehensive literature available on the non-axonemal functions of DNAH9, at least to the best of our knowledge, studies focusing on this area may provide promising results.

Considering all these findings, it appears that the DNAH9 gene may play a role in neoplastic cells, albeit a regulatory rather than a driver role. Without any doubt, further studies focused on DNAH9 and functionally-related genes are warranted for a clearer interpretation. In this manner, the potential of this SNP and other variations of the DNAH9 gene for clinical applications can be fully elucidated.
In the present study, apart from the findings regarding DNAH9, the frequencies of MORN2 rs3099950, PTCRA rs9471966, ANKRD35 rs11579366 and MAGEC1 rs176037 did not exhibit any statistically significant difference between the two groups.

The MORN2 gene is located on the long arm of chromosome 20 (20q13.12) and encodes a protein that integrates into Junctophilin 2 (36). Junctophilins are junctional membrane complexes that regulate signaling between the cell surface and intracellular ion channels (37). Pathogenic variations in Junctophilin 2 have been shown to be associated with hypertrophic cardiomyopathy (MIM: 613873) (38).

PTCRA resides on 6p21.1 and encodes the alpha-chain precursor of the pre-t-cell receptor (pre-TCR). PTCRA contributes to the formation of pre-TCR and T-cell development (39).

ANKRD35 is located on chromosome 1 (1q21.1). The somatic variations of ANKRD35 have been found in 
melanoma, urinary tract malignancies and hematopoietic neoplasias (40).

MAGEC1 resides on Xq27.2 and encodes melanoma antigen family $\mathrm{C} 1$. MAGEC1 is a member of the MAGE family that expresses antigens in tumor cells (41).

The absence of any significant difference for the MORN2 rs3099950, PTCRA rs9471966, ANKRD35 rs11579366 and MAGEC1 rs176037 SNPs between the two patient groups in the present study, is contradictory to the findings of the study by Lavrov et al (16). In their study, Lavrov et al (16) selected a small sample of 8 patients, four of whom were optimal and four were non-optimal TKI-responsive. In this prospect, this discrepancy may be attributed to the low number of patients in the earlier study by Lavrov et al (16). In addition, although the differences between the study designs have been discussed above, these findings are consistent with the later study by Lavrov et al (31).

Mutations in ABL1 kinase domain are a known mechanism of TKI resistance. The importance of detecting additional variants of this domain is evident. For this reason, the present study sequenced exons 4, 5 and 6 of the ABL1 gene. At the end of the analysis, no novel variant was found in the ABL1 kinase domain. This result may be due to the high conservation of the ABL1 kinase domain. Additionally, the exclusion of patients with known pathogenic variations may have also contributed to this result.

In conclusion, the importance of predicting TKI resistance in patients with CML is evident. The results of the present study have provided further evidence of the importance of the DNAH9 rs1990236 SNP in imatinib resistance. It is clear that further clinical studies on DNAH9 rs1990236, as well as other variants that may alter the function of DNAH9 are required. In addition, other genes with similar biological effects need to be investigated in terms of TKI resistance. There is also a need for further studies with larger sample sizes on the MORN2, PTCRA, ANKRD35 and MAGEC1 variants, as well as their effects on TKI resistance. Given the rapid advances in next-generation sequencing technologies, it may be possible in the future to use larger genetic panels that provide information on millions of variants. This may greatly change patient management.

Further clinical, in vitro and in silico studies in this area may lead to the further elucidation of the pathophysiology of CML and may aid in the development of novel methods for the treatment of patients.

\section{Acknowledgements}

Not applicable.

\section{Funding}

The present study was supported by the Research Fund of the Necmettin Erbakan University (project no. 171218022).

\section{Availability of data and materials}

The datasets used and/or analyzed during the current study are available from the corresponding author on reasonable request.

\section{Authors' contributions}

MSY, LŞ, AGZ, ÖÇ and SD were involved in the design and coordination of the study, and the acquisition of data. MSY, LŞ and AGZ analyzed and interpreted the data. MSY and LŞ conducted statistical analyses and wrote the manuscript. MSY, LŞ and AGZ confirm the authenticity of all the raw data. All authors have read and approved the final manuscript.

\section{Ethics approval and consent to participate}

The present study received approval from Necmettin Erbakan University Meram Medical School Ethics Committee and informed written consent for participation and publication were obtained from the patients (protocol no. 2018/1539).

\section{Patient consent for publication}

Not applicable.

\section{Competing interests}

The authors declare that they have no competing interests.

\section{References}

1. Siegel RL, Miller KD and Jemal A: Cancer statistics, 2019. CA Cancer J Clin 69: 7-34, 2019.

2. Faderl S, Talpaz M, Estrov Z, O'Brien S, Kurzrock R and Kantarjian HM: The biology of chronic myeloid leukemia. N Engl J Med 341: 164-172, 1999.

3. Druker BJ, Guilhot F, O'Brien SG, Gathmann I, Kantarjian H, Gattermann N, Deininger MW, Silver RT, Goldman JM, Stone RM, et al: Five-year follow-up of patients receiving imatinib for chronic myeloid leukemia. N Engl J Med 355: 2408-2417, 2006.

4. Noone AM, Howlader N, Krapcho M, Miller D, Brest A, Yu M, Ruhl J, Tatalovich Z, Mariotto A, Lewis DR et al (eds): SEER Cancer Statistics Review, 1975-2015, National Cancer Institute. Bethesda, MD, 2018. https://seer.cancer.gov/csr/1975_2015/.

5. O'Brien SG, Guilhot F, Larson RA, Gathmann I, Baccarani M, Cervantes F, Cornelissen JJ, Fischer T, Hochhaus A, Hughes T, et al: Imatinib compared with interferon and low-dose cytarabine for newly diagnosed chronic-phase chronic myeloid leukemia. N Engl J Med 348: 994-1004, 2003.

6. Branford S, Fletcher L, Cross NC, Müller MC, Hochhaus A, Kim DW, Radich JP, Saglio G, Pane F, Kamel-Reid S, et al: Desirable performance characteristics for BCR-ABL measurement on an international reporting scale to allow consistent interpretation of individual patient response and comparison of response rates between clinical trials. Blood 112: 3330-3338, 2008.

7. Hochhaus A, Baccarani M, Silver RT, Schiffer C, Apperley JF, Cervantes F, Clark RE, Cortes JE, Deininger MW, Guilhot F, et al: European LeukemiaNet 2020 recommendations for treating chronic myeloid leukemia. Leukemia 34: 966-984, 2020.

8. Apperley JF: Part I: Mechanisms of resistance to imatinib in chronic myeloid leukaemia. Lancet Oncol 8: 1018-1029, 2007.

9. Volpe G, Panuzzo C, Ulisciani S and Cilloni D: Imatinib resistance in CML. Cancer Lett 274: 1-9, 2009.

10. Mitchell R, Hopcroft LEM, Baquero P, Allan EK, Hewit K, James D, Hamilton G, Mukhopadhyay A, O'Prey J, Hair A, et al: Targeting BCR-ABL-independent TKI resistance in chronic myeloid leukemia by mTOR and autophagy inhibition. J Natl Cancer Inst 110: 467-478, 2018.

11. Zheng Q, Cao J, Hamad N, Kim HJ, Moon JH, Sohn SK, Jung CW, Lipton JH and Kim DD: Single nucleotide polymorphisms in apoptosis pathway are associated with response to imatinib therapy in chronic myeloid leukemia. J Transl Med 14: 82, 2016. 
12. Ng KP, Hillmer AM, Chuah CT, Juan WC, Ko TK, Teo AS, Ariyaratne PN, Takahashi N, Sawada K, Fei Y, et al: A common BIM deletion polymorphism mediates intrinsic resistance and inferior responses to tyrosine kinase inhibitors in cancer. Nat Med 18: 521-528, 2012.

13. Ko TK, Chin HS, Chuah CT, Huang JW, Ng KP, Khaw SL, Huang DC and Ong ST: The BIM deletion polymorphism: A paradigm of a permissive interaction between germline and acquired TKI resistance factors in chronic myeloid leukemia. Oncotarget 7: 2721-2733, 2016.

14. Kim T, Tyndel MS, Zhang Z, Ahn J, Choi S, Szardenings M, Lipton JH, Kim HJ and Kim Dong Hwan D: Exome sequencing reveals DNMT3A and ASXL1 variants associate with progression of chronic myeloid leukemia after tyrosine kinase inhibitor therapy. Leuk Res 59: 142-148, 2017.

15. Loscocco F, Visani G, Galimberti S, Curti A and Isidori A BCR-ABL independent mechanisms of resistance in chronic myeloid leukemia. Front Oncol 9: 939, 2019.

16. Lavrov AV, Chelysheva EY, Smirnikhina SA, Shukhov OA, Turkina AG, Adilgereeva EP and Kutsev SI: Frequent variations in cancer-related genes may play prognostic role in treatment of patients with chronic myeloid leukemia. BMC Genet 17 (Suppl 1): S14, 2016.

17. McGowan-JordanJ,Simons A and SchmidM(eds): AnInternational System for Human Cytogenomic Nomenclature (2016). Karger, 2016. https://www.karger.com/Book/Home/271658.

18. Solé X, Guinó E, Valls J, Iniesta R and Moreno V: SNPStats: A web tool for the analysis of association studies. Bioinformatics 22 : 1928-1929, 2006.

19. Quintás-Cardama A, Kantarjian HM and Cortes JE: Mechanisms of primary and secondary resistance to imatinib in chronic myeloid leukemia. Cancer Control 16: 122-131, 2009.

20. Fassad MR, Shoemark A, Legendre M, Hirst RA, Koll F, le Borgne P, Louis B, Daudvohra F, Patel MP, Thomas L, et al: Mutations in outer dynein arm heavy chain DNAH9 cause motile cilia defects and situs inversus. Am J Hum Genet 103: 984-994, 2018.

21. Carithers LJ, Ardlie A, Kristin A, Branton PA, Britton A, Buia SA, Compton CC, DeLuca DS, Peter-Demchok J, Gelfand ET, et al: A novel approach to high-quality postmortem tissue procurement: The GTEx Project. Biopreserv Biobank 13: 311-319, 2015.

22. Donner I, Katainen R, Tanskanen T, Kaasinen E, Aavikko M, Ovaska K, Artama M, Pukkala E and Aaltonen LA: Candidate susceptibility variants for esophageal squamous cell carcinoma. Genes Chromosomes Cancer 56: 453-459, 2017.

23. Shah SP, Roth A, Goya R, Oloumi A, Ha G, Zhao Y, Turashvili G, Ding J, Tse K, Haffari G, et al: The clonal and mutational evolution spectrum of primary triple-negative breast cancers. Nature 486: 395-399, 2012.

24. Gruel N, Benhamo V, Bhalshankar J, Popova T, Fréneaux P, Arnould L, Mariani O, Stern MH, Raynal V, Sastre-Garau X, et al: Polarity gene alterations in pure invasive micropapillary carcinomas of the breast. Breast Cancer Res 16: R46, 2014.

25. Spinelli R, Pirola A, Redaelli S, Sharma N, Raman H, Valletta S, Magistroni V, Piazza R and Gambacorti-Passerini C: Identification of novel point mutations in splicing sites integrating whole-exome and RNA-seq data in myeloproliferative diseases. Mol Genet Genomic Med 1: 246-259, 2013.

26. Kusakabe M, Kutomi T, Watanabe K, Emoto N, Aki N, Kage H, Hamano E, Kitagawa H, Nagase T, Sano A, et al: Identification of G0S2 as a gene frequently methylated in squamous lung cancer by combination of in silico and experimental approaches. Int J Cancer 126: 1895-1902, 2010.
27. Gao J, Xi L, Yu R, Xu H, Wu M and Huang H: Differential mutation detection capability through capture-based targeted sequencing in plasma samples in hepatocellular carcinoma. Front Oncol 11: 596789, 2021.

28. Karczewski KJ, Francioli LC, Tiao G, Cummings BB, Alföldi J, Wang Q, Collins RL,Laricchia KM, Ganna A,Birnbaum DP, et al: The mutational constraint spectrum quantified from variation in 141,456 humans. Nature 581: 434-443, 2020.

29. Sim NL, Kumar P, Hu J, Henikoff S, Schneider G and Ng PC: SIFT web server: Predicting effects of amino acid substitutions on proteins. Nucleic Acids Res 40 (Web Server Issue): W452-W457, 2012

30. Quang D, Chen Y and Xie X: DANN: A deep learning approach for annotating the pathogenicity of genetic variants. Bioinformatics 31: 761-763, 2015.

31. Lavrov AV, Chelysheva EY, Adilgereeva EP, Shukhov OA, Smirnikhina SA, Kochergin-Nikitsky KS, Yakushina VD, Tsaur GA, Mordanov SV, Turkina AG and Kutsev SI: Exome, transcriptome and miRNA analysis don't reveal any molecular markers of TKI efficacy in primary CML patients. BMC Med Genomics 12 (Suppl 2): S37, 2019.

32. Campbell V and Copland M: Hedgehog signaling in cancer stem cells: A focus on hematological cancers. Stem Cells Cloning 8: 27-38, 2015.

33. Singh M, Chaudhry $\mathrm{P}$ and Merchant AA: Primary cilia are present on human blood and bone marrow cells and mediate Hedgehog signaling. Exp Hematol 44: 1181-1187.e2, 2016.

34. Colantonio JR, Bekker JM, Kim SJ, Morrissey KM, Crosbie RH and Hill KL: Expanding the role of the dynein regulatory complex to non-axonemal functions: association of GAS11 with the Golgi apparatus. Traffic 7: 538-548, 2006.

35. Miles RR, Crockett DK, Lim MS and Elenitoba-Johnson KSJ: Analysis of BCL6-interacting proteins by tandem mass spectrometry. Mol Cell Proteomics 4: 1898-1909, 2005.

36. Matsushita Y, Furukawa T, Kasanuki H, Nishibatake M, Kurihara Y, Ikeda A, Kamatani N, Takeshima H and Matsuoka R: Mutation of junctophilin type 2 associated with hypertrophic cardiomyopathy. J HumGenet 52: 543-548, 2007.

37. Takeshima H, Komazaki S, Nishi M, Iino M and Kangawa K: Junctophilins: A novel family of junctional membrane complex proteins. Mol Cell 6: 11-22, 2000.

38. Landstrom AP, Weisleder N, Batalden KB, Bos JM, Tester DJ, Ommen SR, Wehrens XH, Claycomb WC, Ko JK, Hwang M, et al: Mutations in JPH2-encoded junctophilin-2 associated with hypertrophic cardiomyopathy in humans. J Mol Cell Cardiol 42: 1026-1035, 2007.

39. Aifantis I, Borowski C, Gounari F, Lacorazza HD, Nikolich-Zugich $\mathrm{J}$ and von Boehmer H: A critical role for the cytoplasmic tail of pT-alpha in T lymphocyte development. Nat Immunol 3: 483-488, 2002.

40. Tate JG, Bamford S, Jubb HC, Sondka Z, Beare DM, Bindal N, Boutselakis H, Cole CG, Creatore C, Dawson E, et al: COSMIC: The catalogue of somatic mutations in cancer. Nucleic Acids Res 47(D1): D941-D947, 2019.

41. Lucas S, De Smet C, Arden KC, Viars CS, Lethé B, Lurquin C and Boon T: Identification of a new MAGE gene with tumor-specific expression by representational difference analysis. Cancer Res 58: 743-752, 1998. International (CC BY-NC-ND 4.0) License. 